\title{
Antidiabetic and Non-specific Immunomodulatory Effects of Vitamin C and Levamisole in Streptozotocine-Induced Diabetic Male Wistar Rats
}

\author{
Aremu, Abdulfatai ${ }^{1}$, Akorede Ganiu Jimoh ${ }^{1}$, Olatunji Omobolanle Aishat ${ }^{1}$, Ahmed \\ Olayiwola Akeem ${ }^{2}$, Raufu Ibrahim Adisa ${ }^{2}$, Afisu Basiru ${ }^{3}$ and Suleiman Kolawole Yusuf ${ }^{3}$ \\ ${ }^{1}$ Department of Veterinary Pharmacology and Toxicology, University of Ilorin, \\ Ilorin, Kwara State, Nigeria. \\ ${ }^{2}$ Department of Veterinary Microbiology, University of Ilorin, Ilorin, Kwara State, Nigeria. \\ ${ }^{3}$ Department of Veterinary Physiology and Biochemistry, University of Ilorin, \\ Ilorin, Kwara State, Nigeria.
}

Accepted November, 2021 and Published December, 2021

\begin{abstract}
Diabetes mellitus is a metabolic disease characterized by absolute or relative deficiencies in insulin secretion associated with chronic hyperglycaemia and disturbances of carbohydrate, lipid and protein metabolism. Diabetes as a metabolic disease has ability to decrease immune system due to oxidative stress. This study was aimed at assessing the antidiabetic and modulatory effect of levamisole and vitamin $\mathrm{C}$ on $\mathrm{CD}^{+}$count, weight changes, haematology, serum chemistry and histopathology alteration of streptozocine induced diabetic rats. Experimental rats were allotted to groups (1-7); 1 (control); 2 (Diabetic control); 3 (Levamisole); 4 (Vitamin C); 5 (Metformin); 6 (vitamin $C$ and levamisole); 7 (Vit C, levamisole and metformin). Diabetes was induced using streptozotocine at $65 \mathrm{mg} / \mathrm{kg}$ administered intraperitoneally. Blood glucose level was monitored using a rapid test strip and glucometer. The CD4+ count was reduced in all the treated diabetic rats when compared with both the two untreated controls. The weights in groups 2, 3, 6 and 7 reduced significantly $(P<0.05)$ while group 4 showed steady weight initially but later reduced significantly $(P<0.05)$ when compared to normoglycaemic control. The haematology showed that all treated groups were not significant to all blood parameters $(p>0.05)$ when compared with the control. The serum chemistry followed the same trend showing a non-significant increase $(p>0.05)$ when compared with the control. Histopathology showed various histoarchitectural changes in the heart, kidney and liver especially in untreated diabetic rats and rats treated with levamisole. Conclusively, the modulatory effect of both levamisole and vitamin $C$ is considerable low in diabetic treated rats when compared to normoglycaemic untreated rats.
\end{abstract}

Keywords: Vitamin C; Levamisole; Streptozotocine and Male Wistar rats

*Corresponding author: 


\section{INTRODUCTION}

Diabetes mellitus is a metabolic disease that is characterized by absolute or relative deficiencies in insulin secretion or insulin action associated with chronic hyperglycemia and disturbances of carbohydrate, lipid and protein metabolism [1].

It may also be said to be a heterogeneous metabolic disorder that can be categorized by the presence of high glucose levels due to impairment of insulin secretion or defective insulin action [2]. It is characterized by impaired glucose metabolism which leads to retinopathy, nephropathy, neuropathy, brain-microinfarcts and other complications [3]

Diabetes mellitus can be broadly classified into Type 1, Type 2 and Type 3. Type 1 diabetes is primarily a result of pancreatic beta cells destruction with a deficiency in insulin which is prone to ketoacidosis and may include cases of the autoimmune process [4]. Type 2 is due to insulin resistance with relative insulin deficiency to a predominantly secretory defect and ketosis is not common while type 3 (gestational diabetes) is a result of glucose intolerance with first recognition during pregnancy [4].

Diabetes is diagnosed using simple methods with the use of test strips on the glycemic blood. [5]. A fasting plasma level of glucose of $7.0 \mathrm{mmol} / 1$ correlates closely with 2 hours plasma glucose value of $11.1 \mathrm{mmol} / 1$ in a $75 \mathrm{~g}$ oral glucose tolerance test(OGTT).

Most experiments using Streptozotocin (STZ) induced diabetic rats have followed the course of the condition for 4-6 weeks, sometimes 8 weeks. However, few studies have extended their measurement period to 24 weeks which is essential to study the mechanisms of the chronic problems of diabetes such as neuropathy [6], retinopathy and nephropathy [7].

Cells of the immune system can be divided into two groups generally which are innate and adaptive. Innate immunity includes granulocytes, mast cells, macrophages and dendritic cells [8]. Both macrophages and dendritic are called antigenpresenting cells (APC) and both classes present and process pathogen peptides on a receptor called the major histocompatibility complex class-II (MHCII). Antigen-presenting cell (APC) comes in contact and activate lymphocyte within the lymphoid tissue, this occurs through the engagement of the MHC-II with the T cell receptor (TCR). T cells are divided into two groups expressing either CD4 or CD8 receptors which play a role in the synapse in the formation of immune synapse between TCR and MHC of lymphocyte and target cells respectively. CD4 cells are activated in the presence of IL-12 and IFN which result in the induction of inflammation and support the function of macrophages and CD8 to kill pathogens. Type 1 diabetes is associated with an autoimmune process resulting from self-reactive CD4 and CD8. T-cells which is followed by massive destruction of immune-mediated pancreatic beta cells of islet of Langerhans [4] which is characteristic of various symptoms including urination, excessive thirst and hunger as a result of inadequate insulin [5]. Three conventional therapeutics used for type 1 diabetes that is currently available includes; insulin therapy, pancreas transplantations and immunotherapy and for this reason; this study is aimed at assessing the non-specific immuno-modulatory effect of vitamin $\mathrm{C}$ and levamisole in streptozocine induced diabetic rats.

\section{MATERIALS AND METHODS \\ Drugs}

Levamisole, metformin and vitamin $\mathrm{C}$ were obtained from a reputable pharmaceutical company in Ilorin, Kwara State, Nigeria.

\section{Experimental Animals}

Twenty-eight male Wistar rats weighing between 120-180 g were used for this experiment. The animals were acquired from the Department of Veterinary Pharmacology and Toxicology, University of Ilorin. 


\section{Animal Management}

Animals were handled humanely and they were fed with vital feed grower pellet and water was provided ad libitum. The animals were kept at the animal house of the Department of Veterinary Pharmacology and Toxicology, University of Ilorin. They were allowed to acclimatize for two weeks before the onset of the experiment.

\section{Induction of Diabetes}

Streptozotocin at the dosage of $65 \mathrm{mg} / \mathrm{kg}$ was used to induce diabetes intraperitoneally and diabetes was confirmed after 2-3 days using fine test glucometer and strip Diagnostic Limited, United Kingdom).

\section{Drugs treatment}

Rats with diabetes in various groups were treated with nonspecific immunostimulants drugs such as levamisole and vitamin C. Metformin was used as conventional anti-diabetic drugs.

\section{Experimental designs}

Each group has ( $\mathrm{n}=4$ rats)

Table 1. Experimental design

\begin{tabular}{ll}
\hline Groups & Description \\
\hline $\mathbf{1}$ & Non-diabetic and untreated (negative control) \\
$\mathbf{2}$ & Diabetic and untreated (positive control) \\
$\mathbf{3}$ & Diabetic treated with levamisole alone \\
$\mathbf{4}$ & Diabetic treated with vitamin C alone \\
$\mathbf{5}$ & Diabetic treated with metformin alone \\
$\mathbf{6}$ & Diabetic treated with vitamin C and levamisole \\
$\mathbf{7}$ & Diabetic treated with vitamin C, levamisole and \\
& metformin \\
\hline
\end{tabular}

\section{Determination of Fasting Glucose}

\section{Concentration}

Blood from diabetic rats was drawn following medial cantus venipuncture. A drop of blood was placed on a fine test strip (diabetes test strip) which was subsequently read using FineTest glucometer ${ }^{\circledR}$ (Auto-coding premium, UK).

\section{Determination of Body Weight}

Bodyweight was determined for each experimental group every week using the convectional weighing scale (Golden Mettler, USA).

\section{Determination of Feeding and Drinking Rate}

Feeding and water intake of both the treated and control group was measured on daily basis using a weighing scale and measuring cylinder. Feeding and drinking habit was determined using the standard method [8]

\section{Haematological Parameters}

The blood samples collected in the heparinized bottles were immediately sent for analysis for full blood count (FBC), packed cell volume (PCV), haemoglobin concentration $(\mathrm{Hb})$ and total white blood cell count (WBC). red blood cell count (RBC), MCV, MCH, MCHC and differential white blood counts (lymphocytes, monocytes, basophils and eosinophils) using Automatic Haematology Analyzer (HA6000 Auto Haematology Analyzer, China). 
Measurement of Serum Biochemical Indices The Serum chemistry analyses were carried out using commercial test kits Biorex ${ }^{\circledR}$ (Diagnostic Limited, United Kingdom) total protein, alanine aminotransferase (ALT), aspartate aminotransferase (ASP), alkaline phosphatase (ALP), creatinine, albumin, globulin and total bilirubin.

\section{Immunological Biomarkers}

The immunological biomarker CD4 was assayed from serum using flow cytometry. The data were acquired and analyzed using a BD LSR II Flow Cytometer. All antibodies were obtained from reliable sources. Isotype controls and fluorescence-minus-one (FMO) controls were used to identify gating boundaries. The CD4 lymphocytes were estimated using a flow cytometer cell counter (Partec, Germany 2010).

\section{Animal Sacrifice}

All the experimental animals were sacrificed after treatment and organs such as the brain liver and kidney were harvested for histopathology.

\section{Histopathological Procedures}

After harvesting the brain, liver and kidney from the rats, the organs were promptly and adequately treated with $10 \%$ formaldehyde (fixation) to preserve their structure and molecular composition. After fixation, a piece of each of the organs was dehydrated by bathing it successively in a graded mixture of ethanol and water $(70-100 \%)$. The ethanol was replaced with a solvent miscible with the embedding medium. As the tissues were infiltrated with xylene, they became transparent (clearing). The impregnated tissue by xylene was placed in melted paraffin in an oven, maintained at $58-60^{\circ} \mathrm{C}$ (embedding). The heat caused the solvent to evaporate and the spaces within the tissues were filled with paraffin. The tissue together with its impregnating paraffin was hardened after removal from the oven. The sections $(5 \mu \mathrm{m})$ were then floated on water and transferred to a glass slide, and stained with haematoxylin and eosin stains. The slides were viewed under a light microscope with magnification X100.

\section{Data Analysis}

All data generated were expressed as mean \pm $\mathrm{SD}$. The differences between the groups were analysed by one-way analysis of variance (ANOVA) followed by Dunnet's posthoc multiple comparison test using Graphpad Prism 5.03 statistical package, San Diego, Califonia, U.S.A (www.Graphpad.Com). P-value was considered significant at $\mathrm{P} \leq 0.05$

\section{RESULTS}

Figure 1 below showed the result of CD-4 count in various treated groups. It shows a significant decrease when compared to diabetic control.

\section{CD-4 COUNT}

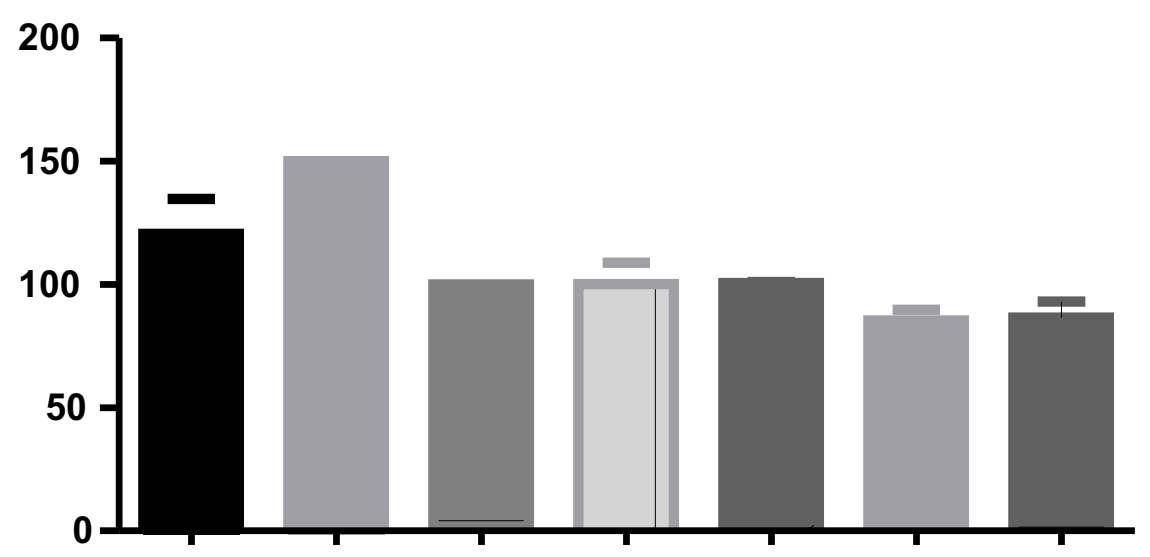

\section{GROUPS}

Figure 1: Effects of immunomodulatory drugs on the CD-4 count of diabetic rats 
Effects of Immunomodulatory Drugs on the Weight of Diabetic Rats

The mean weight was measured on weekly basis and compared with the control. The weights of rats in groups 2, 3, 6 and 7 reduced significantly $(\mathrm{P}<0.05)$ across weeks 2 and 3 when compared with control. Thus, rats in group 4 showed steady weight in the second week but reduced significantly $(\mathrm{P}<0.05)$ in the third week when compared with the control. Then rats in group 5 (Vitamin $\mathrm{C}$ treatment) were not significant $(\mathrm{P}>0.05)$ when compared with control as stated in Table 2 .

Table 2: Modulatory effect of Vitamin C and Levamisole on body weight of diabetic rats

\begin{tabular}{|c|c|c|c|c|c|c|c|}
\hline Grp/wt & 1 & 2 & 3 & 4 & 5 & 6 & 7 \\
\hline Wk 1 & $209 \pm 10.08$ & $201.50 \pm 13.03$ & $190.50 \pm 30.03$ & $195.30 \pm 5.03$ & $204.3 \pm 16.38$ & $206.30 \pm 9.17$ & $180.80 \pm 2.50$ \\
\hline Wk 2 & $219.30 \pm 17.15$ & $186.00 \pm 15.13^{*}$ & $163.50 \pm 29.15^{*}$ & $205.00 \pm 17.15$ & $201.00 \pm 29.81$ & $183.00 \pm 20.58^{*}$ & $173.30 \pm 8.38 *$ \\
\hline Wk 3 & $242.7 \pm 31.21$ & $182.0 \pm 0.00 *$ & $148.50 \pm 26.16^{*}$ & $173.70 \pm 20.13^{*}$ & $205.00 \pm 36.77$ & $177.7 \pm 15.57^{*}$ & $167.0 \pm 33.94 *$ \\
\hline
\end{tabular}

All values are expressed as the mean \pm standard deviation of the mean

* Significantly lower $(\mathrm{P}<0.05)$

Effects of Immunomodulatory Drugs on the the rats was taken before diabetes induction. Fasting Blood Glucose of Diabetic Rats The blood sugar was also measured after The fasting blood sugar of diabetic rats on 3 induction before the treatment over the course weeks treatment with levamisole, vitamin $\mathrm{C}$ of 21 days. The blood sugar was measured on and metformin. The normal blood sugar of all days $0,3,7,10,14$ and 21 as stated in Table 3 .

Table 3: Modulatory effect of Vitamin C and Levamisole on fasting blood glucose of diabetic rats

\begin{tabular}{|c|c|c|c|c|c|c|c|}
\hline Grp/gl & 1 & 2 & 3 & 4 & 5 & 6 & 7 \\
\hline $\begin{array}{l}\text { Normoglyce } \\
\text { mic }\end{array}$ & $98.00 \pm 16.91$ & $99.00 \pm 9.83$ & $104.50 \pm 1748$ & $95.50 \pm 4.79$ & $102.30 \pm 7.13$ & $101.8 \pm 14.24$ & $102.0 \pm 3.46$ \\
\hline Day 0 & $98.00 \pm 16.91$ & $419.3 \pm 156.4$ & $486.5 \pm 145.6 *$ & $315.3 \pm 202.3$ & $360.0 \pm 244.3$ & $445.3 \pm 1827^{*}$ & $464.8 \pm 124.4 *$ \\
\hline Day 3 & $98.00 \pm 16.91$ & $478.30 \pm 105.40 *$ & $* 437.8 \pm 207.5 *$ & $311.3 \pm 205.6$ & $453.3 \pm 200.9 *$ & $508.3 \pm 1282 *$ & $600.0 \pm 0.00^{*}$ \\
\hline Day 7 & $80.75 \pm 20.98$ & $323.00 \pm 162.4^{*}$ & $133.0 \pm 78.60$ & $75.00 \pm 11.34$ & $144.8 \pm 75.55$ & $210.8 \pm 128.4$ & $205.3 \pm 107.9$ \\
\hline Day 10 & $107.80 \pm 20.45$ & $298.70 \pm 261.50$ & $228.00 \pm 205.20$ & $159.80 \pm 45.48$ & $211.50 \pm 84.50$ & $208.30 \pm 36.50$ & $410.00 \pm 236.60$ \\
\hline Day 14 & $109.00 \pm 47.42$ & $590.50 \pm 13.44^{*}$ & $301.30 \pm 212.00$ & $172.00 \pm 139.90$ & $192.50 \pm 103.60$ & $225.70 \pm 81.73$ & $460.70 \pm 232.70 *$ \\
\hline Day 21 & $103.20 \pm 22.12$ & $580 \pm 11.142 *$ & $220.15 \pm 123.13$ & $153.13 \pm 143.23$ & $156.34 \pm 101.21$ & $190.80 \pm 67.23$ & $220.34 \pm 186.62 *$ \\
\hline \multicolumn{8}{|c|}{ All values are expressed as the mean \pm standard deviation of the mean: } \\
\hline * Significar & ntly lower $(\mathrm{P}<0$. & & & & & & \\
\hline
\end{tabular}


Effect of Immunomodulatory Drugs on Haematology of Diabetic Rats

All treated rats were not significant to $\mathrm{PCV}$, RBC, WBC, neutrophils, lymphocyte, monocyte, haemoglobin, $\mathrm{MCH}, \mathrm{MCHC}$
( $>0.05)$ when compared with the control. Mean corpuscular volume was significantly $(\mathrm{P}<0.05)$ reduced in group 6 (rat treated with vitamin $\mathrm{C}$ and levamisole) when compared with the control as indicated in Table 4.

Table 4: Modulatory effect of Vitamin $\mathrm{C}$ and Levamisole on haematological profile of diabetic rats

\begin{tabular}{|c|c|c|c|c|c|c|c|}
\hline Grps/values & 1 & 2 & 3 & 4 & 5 & 6 & 7 \\
\hline PCV & $43.25 \pm 2.36$ & $38.50 \pm 0.70$ & $40.50 \pm 6.36$ & $40.75 \pm 2.75$ & $44.00 \pm 5.65$ & $36.67 \pm 3.05$ & $38.00 \pm 0.00$ \\
\hline RBC & $6.20 \pm 0.30$ & $5.70 \pm 0.00$ & $6.04 \pm 0.60$ & $5.96 \pm 0.23$ & $6.56 \pm 0.94$ & $5.65 \pm 0.21$ & $5.78 \pm 0.040$ \\
\hline WBC & $8.80 \pm 2.51$ & $9.79 \pm 0.14$ & $11.15 \pm 0.49$ & $8.20 \pm 2.17$ & $10.80 \pm 2.26$ & $8.43 \pm 1.50$ & $8.25 \pm 1.90$ \\
\hline Neutr & $58.50 \pm 4.10$ & $67.00 \pm 0.00$ & $60.5 \pm 9.19$ & $62.0 \pm 4.30$ & $55.00 \pm 1.40$ & $64.67 \pm 7.50$ & $56.00 \pm 15.50$ \\
\hline Lympho & $40.00 \pm 4.50$ & $33.00 \pm 0.00$ & $38.5 \pm 9.19$ & $36.50 \pm 3.40$ & $43.00 \pm 1.40$ & $34.60 \pm 6.40$ & $44.00 \pm 15.50$ \\
\hline Mono & $1.50 \pm 0.57$ & $0.00 \pm 0.00$ & $1.00 \pm 0.00$ & $2.00 \pm 0.00$ & $2.00 \pm 0.00$ & $2.00 \pm 0.00$ & $0.00 \pm 0.00$ \\
\hline Hg & $12.80 \pm 0.46$ & $11.80 \pm 0.00$ & $12.15 \pm 1.48$ & $12.13 \pm 0.55$ & $13.45 \pm 1.34$ & $11.27 \pm 1.15$ & $11.85 \pm 0.07$ \\
\hline MCH & $20.50 \pm 0.55$ & $20.50 \pm 0.00$ & $20.10 \pm 0.42$ & $20.30 \pm 0.75$ & $20.55 \pm 0.91$ & $19.73 \pm 1.50$ & $20.25 \pm 0.35$ \\
\hline MCV & $69.00 \pm 0.81$ & $66.00 \pm 0.00$ & $66.50 \pm 3.53$ & $68.00 \pm 1.82$ & $66.50 \pm 0.70$ & $64.33 \pm 2.51^{*}$ & $65.50 \pm 0.70$ \\
\hline МСHC & $29.60 \pm 1.11$ & $31.10 \pm 0.00$ & $30.05 \pm 1.06$ & $29.78 \pm 1.711$ & $30.60 \pm 0.84$ & $30.40 \pm 0.56$ & $31.10 \pm 0.00$ \\
\hline PLT & $173.80 \pm 17.90$ & $171.00 \pm 0.00$ & $183.50 \pm 23.33$ & $180.80 \pm 9.53$ & $171.5 \pm 6.36$ & $172.00 \pm 14.18$ & $191.5 \pm 28.99$ \\
\hline
\end{tabular}

All values are expressed as the mean \pm standard deviation of the mean:

* Significantly lower $(\mathrm{P}<0.05)$

Effects of Immunomodulatory Drugs on Serum Chemistry of Diabetic Rats

The results of serum chemistry of all the treated rats were not significant for AST, ALT, ALP, albumin, urea, creatinin and bilirubin $(\mathrm{p}>0.05)$ when compared with the control. Total protein was significantly reduced in group 2 (positive control) $(\mathrm{P}<0.05)$ when compared with the negative control as stated in Table 5 
Table 5: Modulatory effect of Vitamin C and Levamisole on biochemical profile of diabetic rats

\begin{tabular}{lccccccc}
\hline Grp/values & $\mathbf{1}$ & $\mathbf{2}$ & $\mathbf{3}$ & $\mathbf{4}$ & $\mathbf{5}$ & $\mathbf{6}$ & $\mathbf{7}$ \\
& & & & & & & \\
\hline AST & $6.77 \pm 2.91$ & $2.20 \pm 0.00$ & $6.70 \pm 4.38$ & $4.40 \pm 1.14$ & $4.10 \pm 3.53$ & $6.76 \pm 1.63$ & $2.90 \pm 1.41$ \\
ALT & $4.60 \pm 3.63$ & $4.20 \pm 0.28$ & $7.35 \pm 3.75$ & $5.12 \pm 1.34$ & $6.15 \pm 3.04$ & $5.03 \pm 1.87$ & $4.80 \pm 2.68$ \\
ALP & $15.98 \pm 10.7$ & $3.90 \pm 0.00$ & $14.40 \pm 8.8$ & $11.10 \pm 1.94$ & $14.40 \pm 6.22$ & $10.93 \pm 3.39$ & $11.25 \pm 1.6$ \\
& & & & & & & \\
Total P & $7.25 \pm 1.79$ & $5.00 \pm 0.00^{*}$ & $6.90 \pm 1.27$ & $6.67 \pm 0.47$ & $8.25 \pm 0.49$ & $7.06 \pm 0.57$ & $8.75 \pm 1.06$ \\
& & & & & & & \\
Albumi & $2.40 \pm 0.73$ & $1.50 \pm 0.70$ & $2.30 \pm 0.70$ & $2.35 \pm 0.33$ & $2.35 \pm 0.91$ & $2.00 \pm 0.00$ & $2.60 \pm 0.56$ \\
Urea & $3.30 \pm 1.04$ & $4.90 \pm 0.00$ & $3.40 \pm 0.56$ & $2.85 \pm 0.98$ & $3.15 \pm 0.35$ & $2.53 \pm 1.18$ & $2.55 \pm 0.70$ \\
Creatinin & $73.50 \pm 17.1$ & $89.00 \pm 0.00$ & $81.00 \pm 4.24$ & $70.75 \pm 10.69$ & $83.00 \pm 24.04$ & $55.33 \pm 24.03$ & $60.50 \pm 6.36$ \\
& & & & & & & \\
Bilirubin & $1.67 \pm 0.63$ & $1.00 \pm 0.00$ & $2.20 \pm 0.56$ & $1.75 \pm 0.60$ & $1.75 \pm 0.35$ & $2.25 \pm 0.77$ & $1.85 \pm 0.63$ \\
\hline
\end{tabular}

All values are expressed as the mean \pm standard deviation of the mean:

* Significantly lower $(\mathrm{P}<0.05)$

\section{Histopathology results of Heart}

I

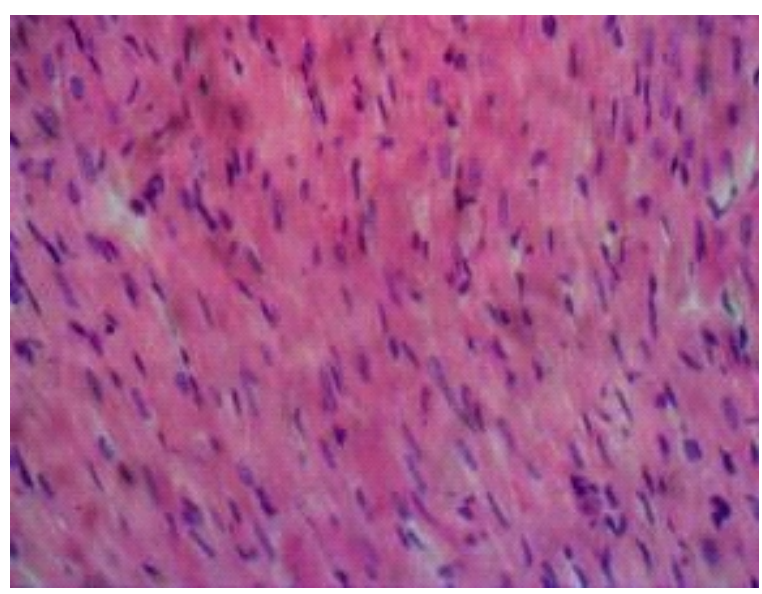

Plate I. The photomicrograph of the heart of rats in group 1 (un-induced and untreated; $\mathrm{H} \& \mathrm{E}$ X40) showed no visible lesion (NVL)
II

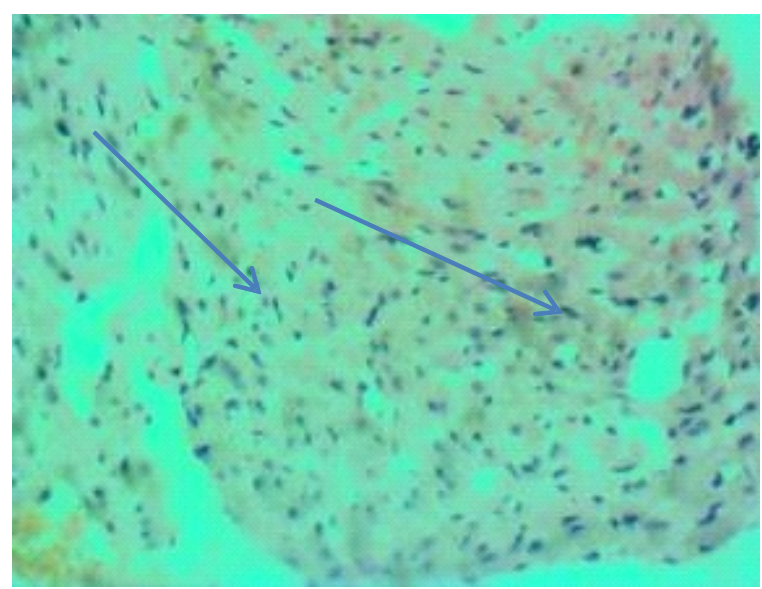

Plate II. The photomicrograph of the heart of diabetic rats in group 2 (induced and untreated H\&E X40) showing myofibre atrophy and loss of striations (arrows) 
III

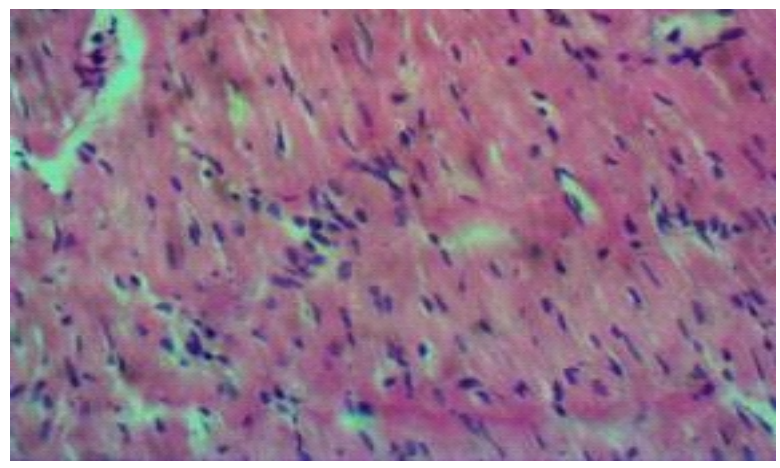

Plate III. The photomicrograph of the heart of diabetic rats in group3 treated with levamisole only (H\&E X40) showed no visible lesion (NVL)

\section{V}

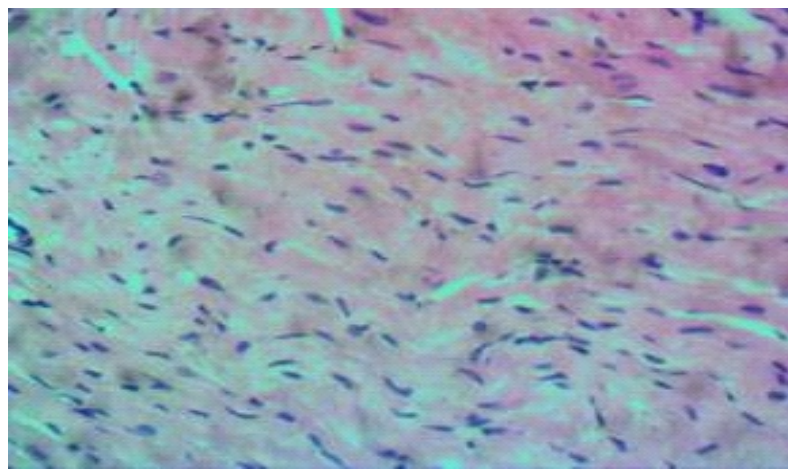

Plate V: The photomicrograph of the heart of diabetic rats in group 5 treated with metformin only (H\&E X40) showed no visible lesion (NVL)

VII

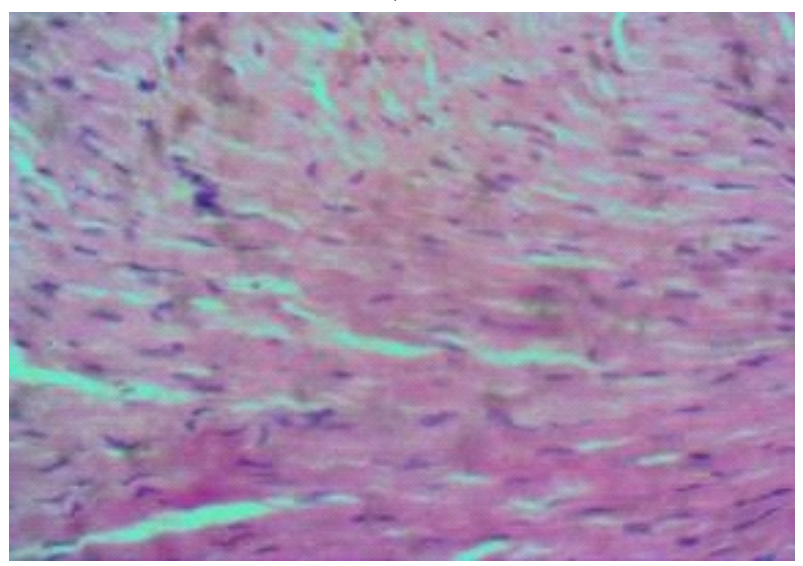

Plate VII: The photomicrograph of the heart of diabetic rats in group 7 treated with levamisole, Vitamin C and metformin (H\&E X40) showed no visible lesion (NVL)
IV

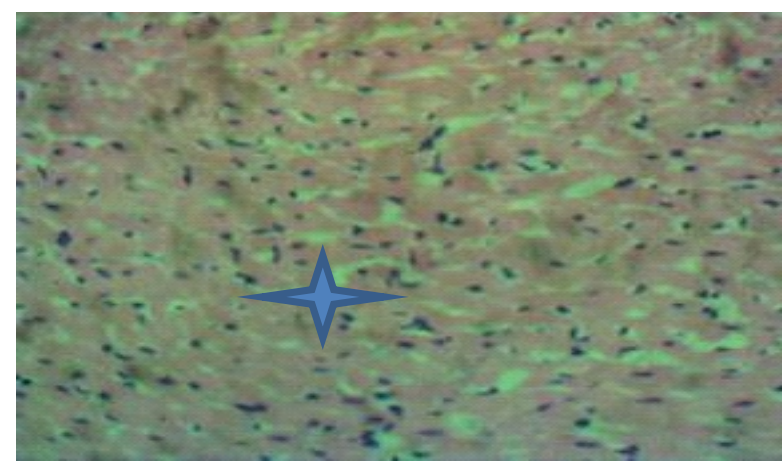

Plate IV. The photomicrograph of the heart of diabetic rats in group 4 treated with vitamin C only (H\&E X40) showed multiple foci of myofiber degeneration

VI

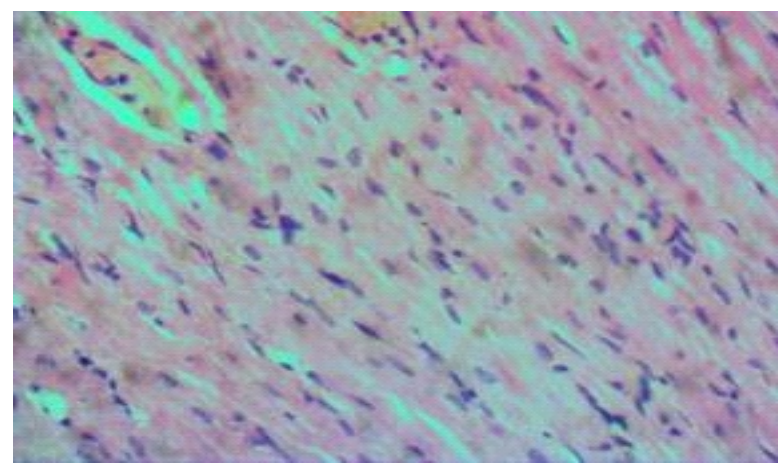

Plate VI: The photomicrograph of the heart of diabetic rats in group 6 , treated with levamisole and Vitamin C (H\&E X40) showed no visible lesion (NVL) 


\section{Histopathology results of Kidneys}

VIII

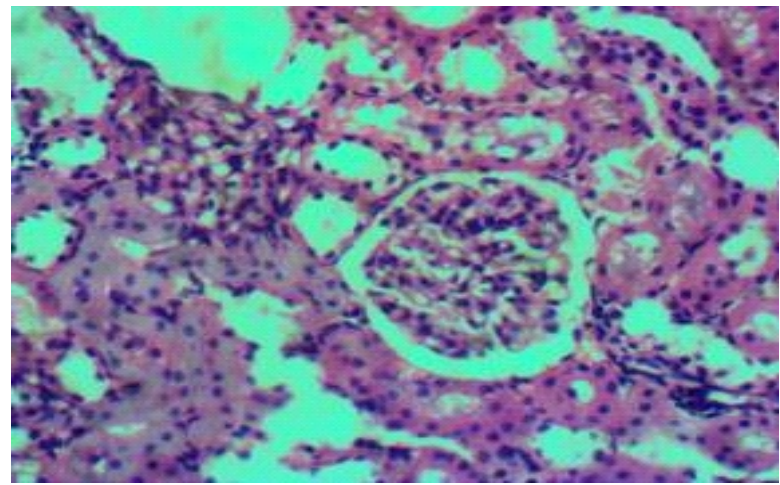

Plate VIII; The photomicrograph of the kidney of rats in group 1 (un-induced and untreated) (H\&E X40) showedno visible lesion (NVL)

$\mathrm{X}$

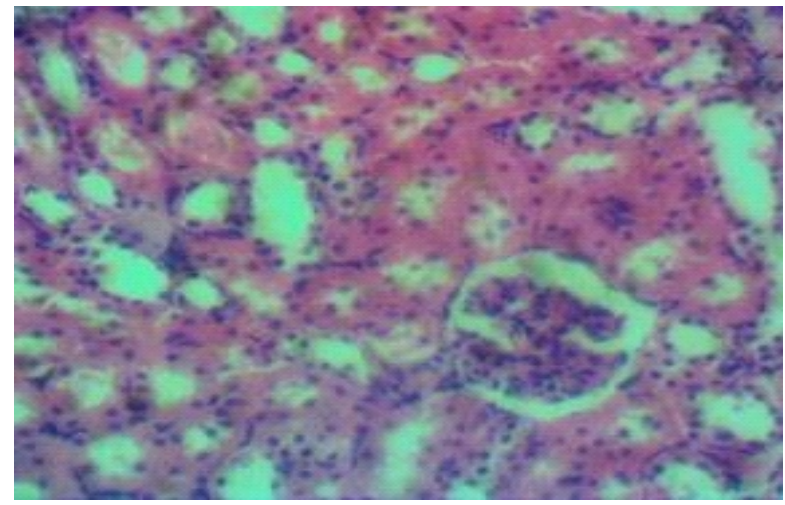

Plate X: The photomicrograph of the kidney of diabetic rats in group 3 treated with levamisole only (H\&EX40) showed no visible lesion (NVL)

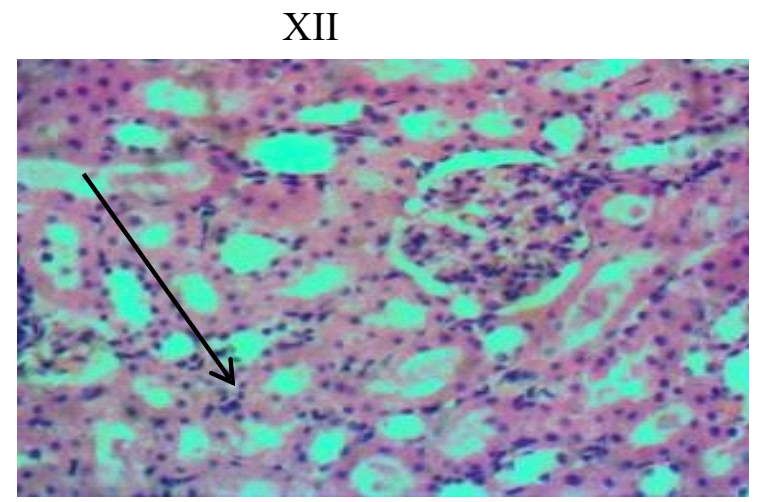

Plate XII: The photomicrograph of the kidney of diabetic rats in group 5 treated with metformin only (H\&E X40) showed a few cellular casts in the tubular lumen (arrow).
IX

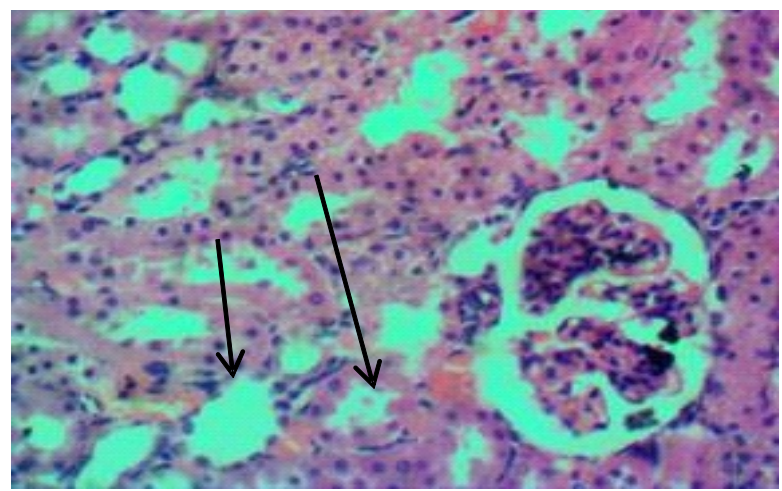

Plate IX: The photomicrograph of the kidney of diabetic rats in group 2 (induced and untreated) (H\&E $\mathrm{X} 40$ ) tubular epithelial necrosis (arrows) and ectasia.

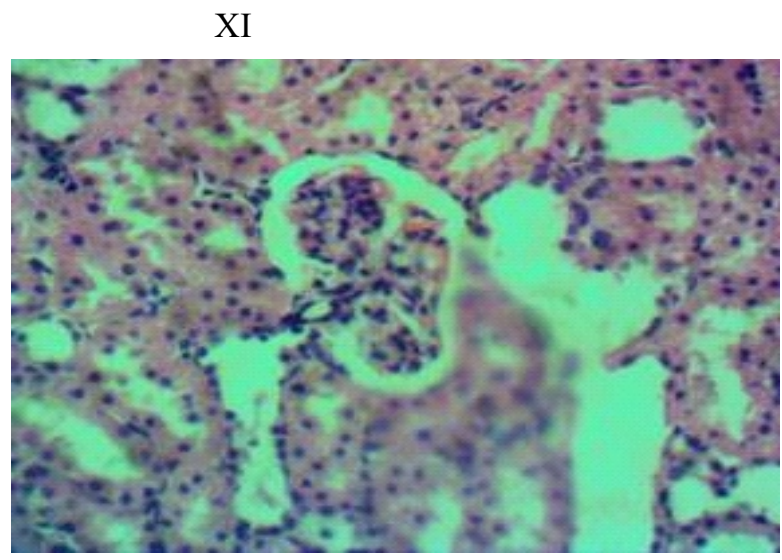

Plate XI: The photomicrograph of the kidney of diabetic rats in group 4 treated with vitamin C only (H\&EX40) showed no visible lesion (NVL).

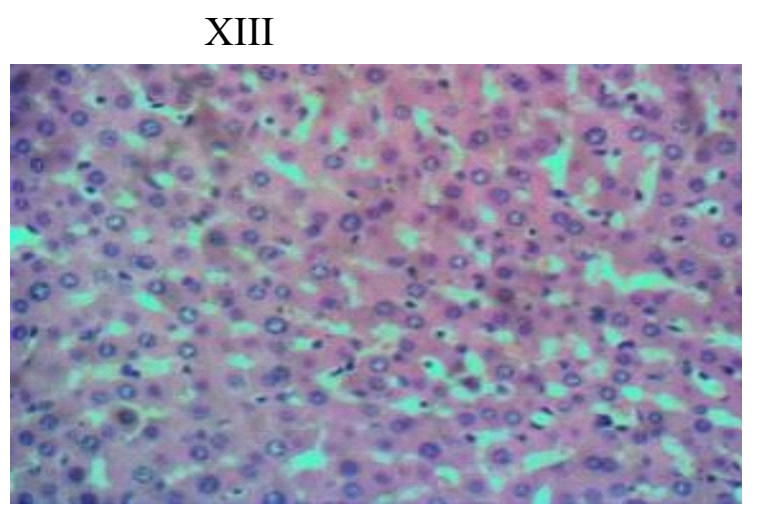

Plate XIII. The photomicrograph of the kidney of diabetic rats in group 6, treated with levamisole and Vitamin C (H\&EX40) showed moderate atrophy of nephrotic cells 


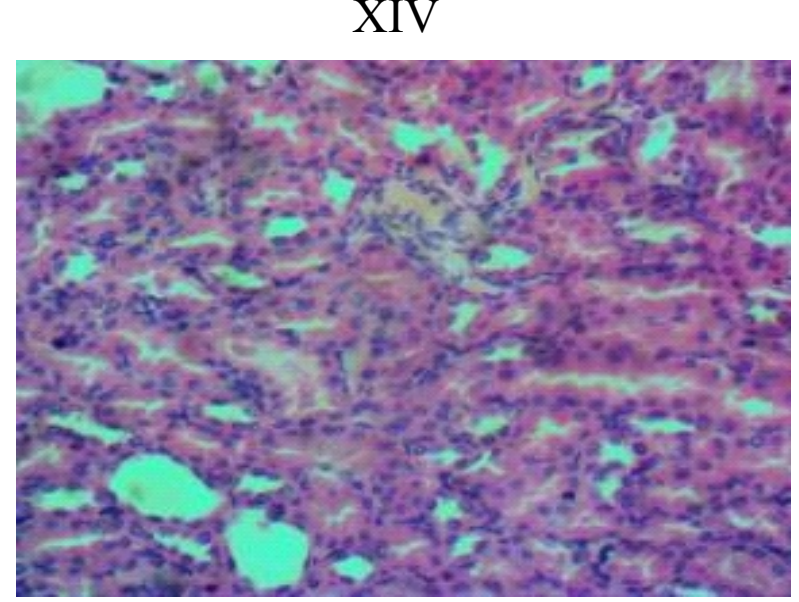

Plate XIV: The photomicrograph of the kidney of diabetic rats in group 7 treated with levamisole, Vitamin $\mathrm{C}$ and metformin (H\&E X40) showed no visible lesion (NVL)

\section{Histopathology results of Liver}

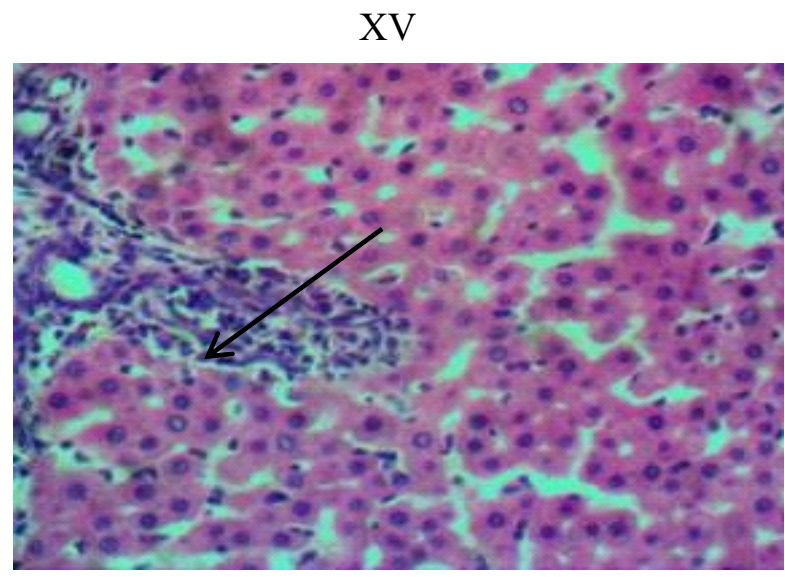

Plate XV: The photomicrograph of the liver of rats in group 1 (un-induced and untreated) (H\&E X40) showed mononuclear cellular infiltrates in the portal areas (arrow).

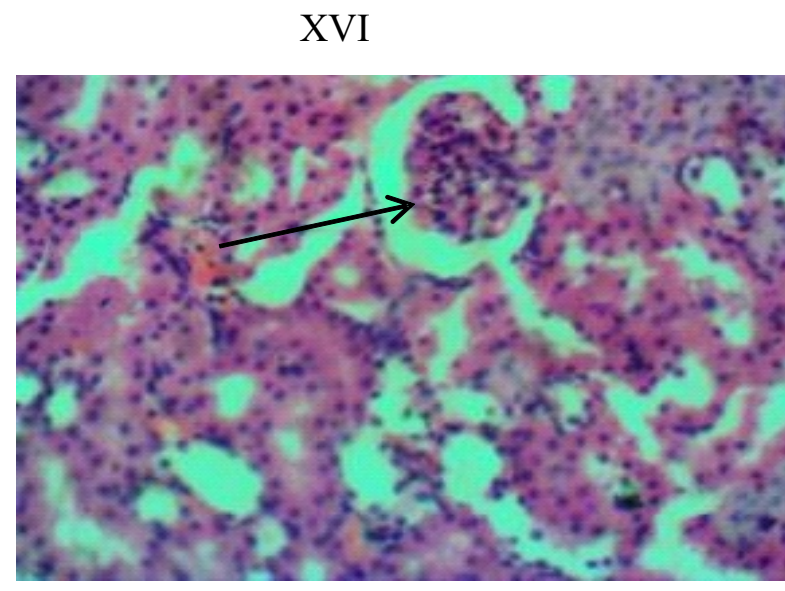

Plate XVI. The photomicrograph of the liver of diabetic rats in group 2 (induced and untreated) (H\&E X40) showed diffuse hepatocellular atrophy and hyperplasia of Kupffer cells (arrows) 
XVII

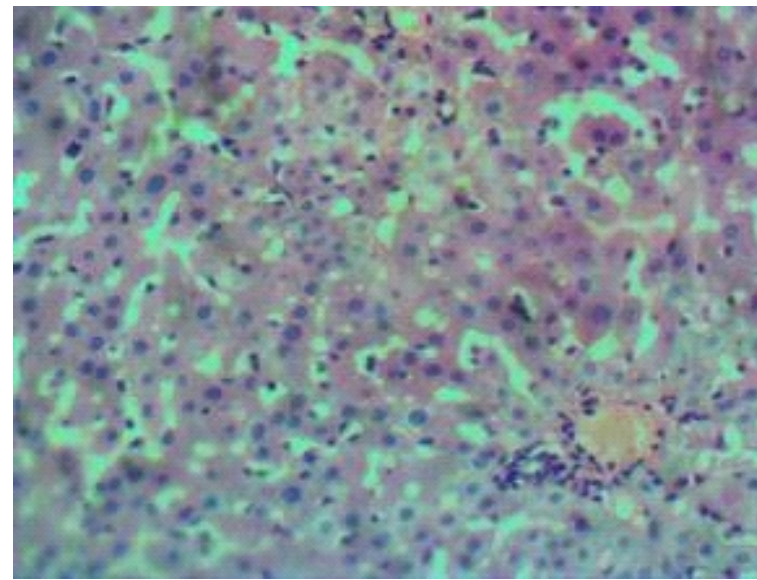

$\backslash$ Plate XVII: The photomicrograph of the liver of diabetic rats in group 3 treated with levamisole only (H\&E X40) showed random hepatocellular degeneration and necrosis with perivascular cellular infiltrate

\section{XIX}

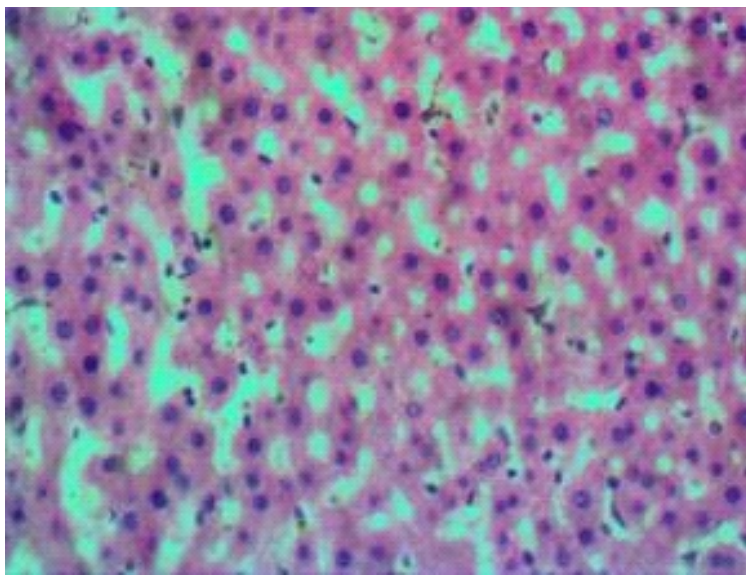

Plate XIX:The photomicrograph of the liver of diabetic rats in group 5 treated with metformin only (H\&E X40) showed moderate diffuse atrophy of hepatocytes.
XVIII

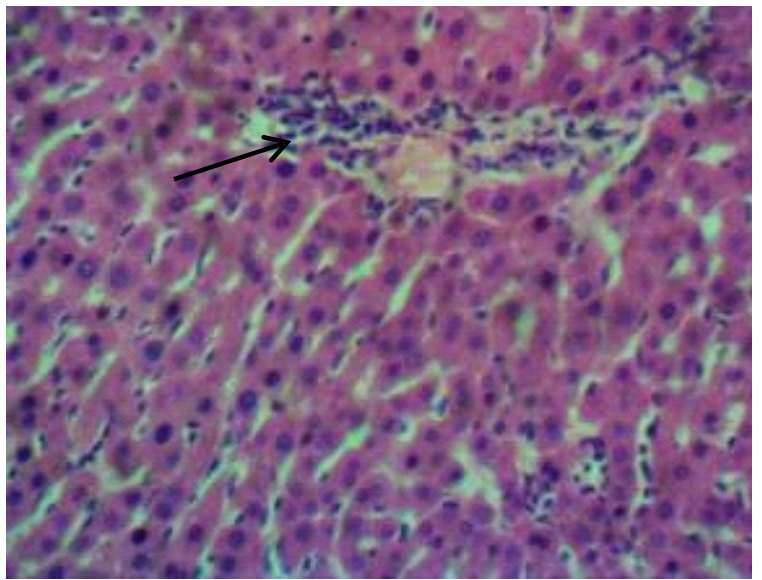

Plate XVIII: The photomicrograph of the liver of diabetic rats in group 4 treated with vitamin $\mathrm{C}$ only (H\&E X40) showed multiple foci of necrotizing hepatitis

\section{XX}

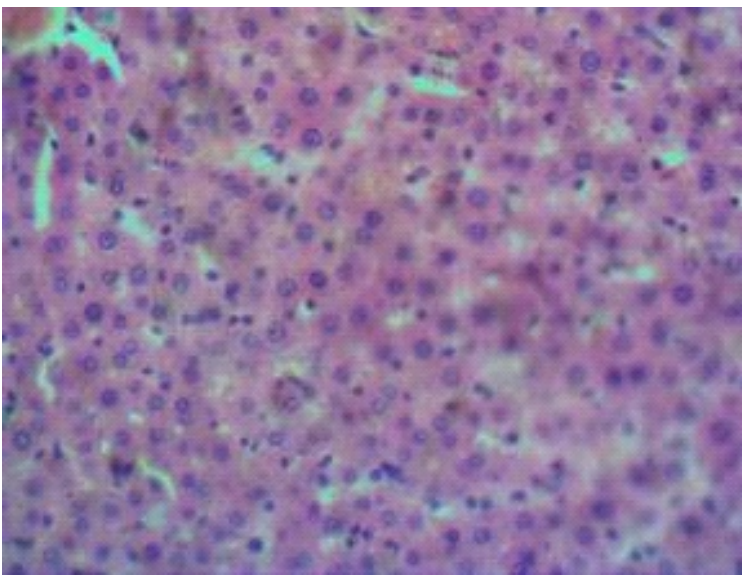

Plate XX. The photomicrograph of the liver of diabetic rats in group 6 , treated with levamisole and Vitamin C (H\&E X40) showed no visible lesion (NVL). 


\section{XXI}

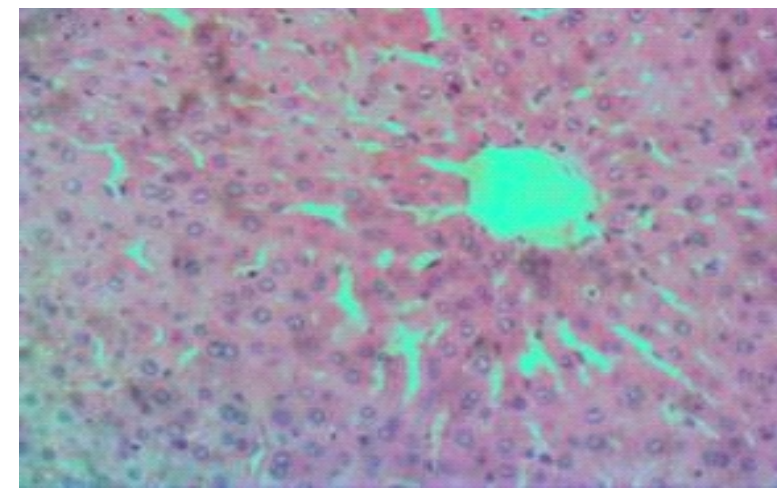

Plate XXI: The photomicrograph of the liver of diabetic rats in group 7 treated with levamisole, Vitamin $\mathrm{C}$ and metformin (H\&E $\mathrm{X} 40$ ) showed no visible lesion (NVL).

\section{DISCUSSION}

The CD4 count is used to measure the number of CD4+ cells in the blood and this is mostly referred to as T-cells which combat infection and play an important role in the immune system. It is mostly used as a marker to check the health status of the immune system, especially in HIV patients. If too many CD4+ cells are lost the immune system will find it difficult to wipe off various infections [20]. The result of this study showed increased CD4+ count in diabetic untreated rats when compared with non-diabetic rats and this may be solely attributed to the inflammatory response to atherosclerotic pathogenesis mostly seen in diabetes. Bloodborne immune cells constitute an important part of atheroma known as the core of which is infiltrated by T-cells and predominantly the CD4+ T-cells [20]. Metabolic breakdown in diabetes usually results in increased circulation and deposition of lipids in arteries thus increasing immune and inflammatory response which eventually leads to an elevation in associated T-cell variant. The outcome of this study showed a reduction in CD4+ count in all the treated groups when compared with both the diabetic and non- diabetic untreated rats. This observation may be attributed to the transient healing effects of all the three drugs subsequently showing their anti-inflammatory properties. Though, the immunomodulatory effects of both vitamin $\mathrm{C}$ and levamisole were not well defined as both drugs showed the same average values. The combined drugs in both groups 6 and 7 showed reduced count when compared to single drugs in groups 3 and 4 .

Diabetes mellitus results in unexplained weight loss that usually occurs unintentionally and this is known to be the warning sign of diabetes [9]. This scenario is attributed to insufficient insulin which prevents the body from getting glucose from the blood into the body cells serving as the source of energy. The body system will mobilize the fatty layers for gluconeogenesis thereby causing a reduction in overall body weight [9].

The result of this study showed that there is a significant reduction in the percentage weight in the normoglycaemic untreated rats across the three weeks but there was a significant improvement in weight gain especially in the group treated with vitamin $\mathrm{C}$ and metformin when compared with the control. The levamisole treated group showed a significant decrease in percentage weight thereby worsening the episode of weight loss. This observation may be attributed to the toxic unwanted effect of the drug. The combinationtreated rats (group 7) showed transiently decreased weight when compared with the untreated group. This outcome was in conformation with various studies that ascertain that both type 1 and type 2 diabetes led to unexplained weight loss [10]. 
Blood glucose levels give a vital and significant clue about managing diabetes which leads to high fasting blood glucose levels to insulin resistance [11]. Blood glucose level between meals offers a pathway into how body glucose is being managed. High levels of fasting blood glucose suggest that the body has been unable to lower the levels of glucose in the blood and at this point either insulin resistance (type 2 diabetes) or inadequate insulin production (type 1) or even both. As with low blood glucose, high blood glucose may also cause loss of consciousness or seizure if left untreated [12]. The outcome of this study showed that the blood glucose of rats in all the groups increased significantly $(\mathrm{P}<0.05)$ at days 0 and 3 when compared with normoglycaemic control. Thus, by day 7 , the only diabetic untreated group increased significantly. Diabetic treated rats showed reduced blood glucose by days 10, 14 and 21 but were not significant $(\mathrm{P}>0.05)$ when compared with non-diabetic rats. All the treated rats showed decreased blood glucose especially those treated with vitamin $\mathrm{C}$ and metformin respectively. This observation conforms with the result of a study from Deakin University who found out that taking $500 \mathrm{mg}$ of Vitamin C twice daily is effective in lowering the blood glucose level in type 2 diabetes [12].

Several haematological changes affecting red blood cells white blood cells and coagulation factors are known to be directly associated with diabetes mellitus [13]. The occurrence of anaemia in diabetes mellitus has been linked to increasing in non-enzymatic glycosylation of red blood cell membrane proteins [14]. The oxidation of these proteins and hyperglycemia in diabetes mellitus leads to an increase in the production of lipid peroxide which subsequently results in hemolysis of red blood cells [15]. The outcome of this study showed a non-significant decreased PCV where all the treated rats showed decreased values except the metformin whose values is slightly higher when compared with non-diabetic rats. The $\mathrm{RBC}$ of vitamin $\mathrm{C}$ and metformin-treated rats increased non-significantly thereby showing their regenerative abilities. Other rats in various groups followed the trend of [15] showing a slight decreased RBC. The WBC, neutrophils, lymphocyte, monocyte and haemoglobin showed non-significant increased values $(p>0.05)$ when compared to nondiabetic rats. It has been reported that the mean corpuscular haemoglobin concentration was higher in diabetes patients while the mean corpuscular volume (MCV) is lower in diabetes [16]. The result of this study follows the same trend with [16] as seen in all the diabatic treated rats showing a non-significant increased MCHC except the vitamin $\mathrm{C}$ treated rats that showed the same trend with the non-diabetic rats. This observation agrees with [16] who reported that MCV decreased non-significantly in diabetic treated rats. Patient with type 2 diabetes mellitus has been reported to have an increased risk of coagulative abnormalities and thromboembolic events. Platelets have a key role and increase adhesions, activations and aggregations due to dysregulation of several signalling pathways and metabolic disturbances as a result of insulin resistance, hyperglycemia and dyslipidemia which are normally seen in diabetes [17]. The result in this study showed a non-significant increase in all the treated rats except metformin and diabetic untreated rats that showed decreased platelet count when compared to non-diabetic rats.

Hyperglycemia is one of the major causes of progressive renal damage and various reports reveal that there exists a strong relationship between the increase in blood glucose level, blood urea and serum creatinine levels. This increase may be due to kidney damage. The 
decrease in kidney function in a diabetic patient may be due to several reasons such as changes in urinary albumin, excretion rate, glomerular filtration rate and glycosylated haemoglobin levels [18]. The result of this study showed that all the treated rats were not significant to albumin, urea, creatinine and bilirubin when compared to non-diabetic rats. Total protein was significantly reduced in diabetic untreated rats (group 2) when compared to all treated rats and non-diabetic rats. Previous studies have shown that type 2 diabetes is associated with a clinical spectrum of liver abnormalities and have shown higher levels of AST, ALT and ALP in many diabetic patients [19]. The outcome of this study showed contradicting views where the diabetic untreated rats and drug combination-treated rats showed a nonsignificant decrease to both AST and ALT. This study further emphasized that all the treated rats were not significant to AST, ALT, ALP, $(p>0.05)$ when compared to non-diabetic rats.

The histopathology result of this study revealed that the heart of diabetic untreated rats had myofiber atrophy and loss of striations showing that the structural organizations of cardiac tissues have been disturbed which subsequently leads to myocardial injury. This observation was consistent with the work of [21] who confirm that diabetic rats showed cardiac histopathological changes. This study further showed that the entire treated rats had no observable lesion on the cardiac tissues except Vitamin $\mathrm{C}$ treated rats that showed multiple foci degeneration of the myofibril. This observation may be due to the aftermath effect of vitamin $C$ on the cardiac tissues.

Diabetes is an epidemic disease and reports have shown that it is the leading cause of most kidney diseases. The photomicrograph of the kidney of untreated diabetic rats revealed tubular epithelial necrosis and ectasia which is a sign of nephron damage. The outcome further emphasizes that the immunomodulatory drugs vitamin $\mathrm{C}$ and levamisole showed no observable lesions but treated with metformin and drug combination showed a few cellular casts in the tubular lumen and moderate atrophy of nephrotic cells. Histopathological changes seen in streptozocine induced diabetes animals has been previously reported [22]. The liver is an insulin-dependent tissue, playing a pivotal role in glucose and lipid homeostasis is known to be severely affected during diabetes. Various studies suggested that the liver of an untreated diabetic patient activates some isoforms of protein kinase $\mathrm{C}$ in tissues and hepatocytes. Mitogen-activated protein kinase will now lead to decrease apoptosis and hyperplasia and finally result in diabetic hepatomegaly [23]. The photomicrograph result of the liver of diabetic untreated rats in this study showed diffuse hepatocellular atrophy and hyperplasia of Kupfer cells. Most of the treated rats (groups 3-5) showed random hepatocellular degeneration and necrosis inexception of groups 6 and 7 that showed no marked lesion. The degeneration observed in the vitamin $\mathrm{C}$ treated group is considerably reduced when compared with the untreated diabetic rats.

\section{Conclusion}

That immunomodulatory effect of both levamisole and vitamin $\mathrm{C}$ is considerable low after 21 days of treatment in diabetic rats judging by the CD4+ count. Vitamin $\mathrm{C}$ has almost the same anti-diabetes effect as metformin as observed in the transient reduction of blood glucose following 21 days of treatment. Vitamin C improves the weight in diabetic rats as observed over the course of 21 days of treatment. The synergistic action of 
both vitamin $\mathrm{c}$ and levamisole is not well promising as observed in the weight and blood glucose reduction following 21 days treatment. Both vitamin $\mathrm{C}$ and levamisole showed improved observation in both haematology and biochemistry parameters when compared to metformin-treated groups. The histopathology of the heart, kidney and liver is adequately improved in all the treated groups when compared with the diabetic control

\section{Acknowledgement}

The authors wish to express their gratitude to Mr Afolabi Adegboye, Mr Moshood Bolaji and Mr Shafi Alada. There was no conflict of interest regarding this work. This work did not receive funding from any individual or organization

\section{REFERENCES}

1. Duckworth, W. C. (2001). Hyperglycemia and cardiovascular disease. Current Atheries. Rep.3, 38-391.

2. America Diabetes Association (2012). Diagnosis and Classification of Diabetes Mellitus. Diabetic Care 35. S64-71.

3. Harris, M. I, Klein, R. and Welborn, T. A. (2002). Onset of NIDDM occurs at least 4-7 yr before clinical diagnosis. Diabetic Care. 15(7):815-819 10.2337/diacare.15.7.815.

4. Amed, S. and Oram R. (2016): MaturityOnset Diabetes of the young. making the right diagnosis to Optimize Treatment. Canadian Journal of Diabetes. 40:449-54.

5. Sacks, D. B, Arnold, M. and Bakris, G. L. (2011). Executive Summary: Guidelines and recommendations for laboratory analysis in the diagnosis and management of diabetes Mellitus. Clinical Chemistry 57:793-8.
6. Cameron, N. E and Cotter, M. A. (2001). Diabetes. Causes and early reduction in autonomic ganglion blood flow in rat. Journal of diabetes Complication. 15: 1982022001.

7. Mifsud, S.A., Allen, T. J., Bertram, J.F., Hulthen, U. L., Kelly, D. J., Cooper, M. E., Wilkinson-Berka, J. L. and Gilbert, R. E. (2001). Podocyte food process broadening in experimental diabetic nephropathy: amelioration with renin-angiotensin blockade. Diabetologia; 44: 878-82.

8. Medina, E. (2009). Neutrophil extracellular traps: A Strategic Tactic to defeat pathogens with potential consequences for the host. Journal of innate Immunity 1(3):176-180.

9. Hossain, P, Kawar, B. and El Nahas, M. (2007). obesity and diabetes in the developing world- a growing challenges. New England Journal of Medicine, 356: 213-215.

10. Karan, S. R, Chathyan, P. and Islam A. (2017). Plasma irisin is elevated in type 2 diabetes and is associated with increased Eselectin levels. Journal of Cardiovascular and Diabetes. 16:147. Doi 10.1186/s12933-017-0627-2.

11. Bonds, D. E, Zaccaro, D.J and Karter, A. J. (2005). Ethnic and racial difference in diabetes care. The insulin resistance atherosclerosis Study. Journal of Diabetes Care. 26 (4): $1040-1046$. Doi.Org/10.2337/Diacare.26.4.1040.

12. Sang, W.S., Aaron, M, Hamby, R. A, Yang, F., Christian, T.S., Haruna, T., Atsushi, T and Jialing, L. (2007). Hypoglycemia, brain energetics and hypoglycemic neuronal death. Glia (Wiley Online library). Doi.Org/10.1002/Glia.20440.

13. Samuel, A. B, Ransford, K., Samuel, O. B., Max, A., Jonathan, K. A. Otchere, A and Margaret, F. (2018). Haematological parameters and lipid profile abnormalities 
among patients with type- 2 diabetes mellitus in Ghana. Lipid health and diseases 17:283.

14. Ige, A. O. and Adewoye, O. E. (2016). Oral magnesium treatment reduces anemia and Levels of inflammatory markers in experimental diabetes. Journal of Diet $\begin{array}{lllllllllll}S & u & p & p & l & e & m & e & n & t & s\end{array}$. Doi.Org/10.1080/19390211.2016.120570 0 .

15. Arun, K. B, Dhanya, R., Syama, H. P., Nisha, P., Sundareesan, T. R., Kumar, S. and Jayamurthy A. (2014). Rutin and quercetin enhance glucose uptake in L6 myotubes under oxidative stress induced by tertiary butyl hydrogen peroxide. Food chemistry. 02.151 Doi.Org/10.1016/J.

16. Mirza, S., Monir, H., Christine, M. Martinez, P. Pino P. Gay, J. L., Rentfro, A., McCormick J. B. and Fisher-Horch S. P. (2011): Type 2-diabetes is associated with elevated levels of TNF-Alpha, IL-6 and adinopectin and low levels of leptin in a population of mexican Americans: a crosssectional study. Journal of Cytology 09.029 Doi.Org/10.1016/.

17. Kim, Y. D., Park, K. G., Lee, Y. S Park, Y., Kim, D., Balachandar, N., Jang, W. G., Cho, W. J., Lee, I., Lee, C. and Cho, H. (2008). Metformin inhibits hepatic gluconeogenesis through AMP-activated protein kinase-dependent regulation of the orphan nuclear receptor SHP. Journal of Diabetes 57(2):306-314 10.2337/db07038.

18. Thomas, C. Chenier, R. M., Cohen, Y. R., Yancey, R., Holmes, M. D. Clinton, H. and Joiner, M. D. (2003). Discordance between $\mathrm{Hba}$ and Fructosamine. Evidence for a glycosylation gap and its relation to diabetic nephropathy. Diabetes Care: 163167. Doi-Org/10.2337/Diacare.26.1.163.

19. Balogun, A. J, Sanyal, A. M., Northup, P. G., Sundaram, V., Fallon, M. B. and Reddy, K. R. (2008). Hypercoagulation and thrombophilia in liver disease. Journal of Thrombocytes and Haemoglobin (Wiley online library). Doi.Org/10.1111/J.15387836.2007.02772.

20. Hansson, M., Christian, T., Anne, M., Mariana, Q., Ane, M., Malin, S. and Daniela, B. (2012). DC-derived IL-18 drives treg differentiation, murine helicobacter pylori-specific immune tolerance and asthma protection. Journal of Clinical Investigation. 10.1172/JC161029.

21. Zar, C. T., Teoh, S. L., Srijit, D. and Zaiton, Z. (2012). Histological changes in the heart and the proximal aorta in experimental diabetic rats fed with piper samentsoum. African Journal of Traditional, complementary and alternative Medicine. 9(3): 396-404. PMID 23983373.

22. Sandhu, K, Randhawa, S. S. and Rajinder, S. B. (2000). clinical and pathological changes in alloxan induced diabetes mellitus alone and in combination with ethylene glycol induced nephropathy in dogs. Journal of Veterinary Pathology, 24:12-15.

23. Herman, C. E, Sanders, R. A, Klaunig, J. E., Hensen, B. and Justin, B. (1999). Decreased apoptosisas a mechanism for hepatomegaly in streptozocin-induced diabetic rats. Journal of Toxicological Science. 50 (1).146-51. 